1989-09-01

\title{
Probabilistic Segmentation of Myocardial Tissue by Deterministic Relaxation
}

\author{
William A. Barrett \\ william_barrett@byu.edu \\ Shawn C. Becker \\ Jerome A. Broekhuijsen
}

Follow this and additional works at: https://scholarsarchive.byu.edu/facpub

Part of the Computer Sciences Commons

\section{Original Publication Citation}

Jerome A. Broekhuijsen, Shawn C. Becker, and William A. Barrett: "Probabilistic Segmentation of Myocardial Tissue by Deterministic Relaxation." IEEE Proceedings of Computers in Cardiology, pp. 99-12, Jerusalem, September, 1989.

\section{BYU ScholarsArchive Citation}

Barrett, William A.; Becker, Shawn C.; and Broekhuijsen, Jerome A., "Probabilistic Segmentation of Myocardial Tissue by Deterministic Relaxation" (1989). Faculty Publications. 738.

https://scholarsarchive.byu.edu/facpub/738 


\title{
PROBABILISTIC SEGMENTATION OF MYOCARDIAL TISSUE BY DETERMINISTIC RELAXATION
}

\author{
Jerome A. Broekhuijsen, Shawn C. Becker, and William A. Barrett \\ Department of Computer Science \\ Brigham Young University \\ Provo, Utah 84602
}

\section{$\underline{\text { Summary }}$}

A recently-developed probabilistic model for automatically segmenting regions of interest in abdominal CT scans has been adapted to the challenging task of segmenting myocardial tissue in Cine CT scans. A system has been implemented on relatively low-cost hardware which performs such segmentations. Special techniques have been developed to improve consistency and accuracy. Early results of testing this new modality are encouraging and promising. Based on observations from experimentation, new directions for future work have been identified.

\section{Motivation}

Automated detection and measurement of myocardial tissue from axial CT images are required in a number of applications including quantification of wall thickening, estimation of myocardial perfusion, and three- and four-dimensional (3D and 4D) visualization of the myocardial surface. When injected contrast is used in conjunction with Cine CT, traditional image processing techniques such as intensity thresholding have been shown to be effective for isolating and visualizing the $4 \mathrm{D}$ endocardial surface. However, threshold segmentation alone appears to be inadequate for definition of the myocardium and epicardial surface due to limitations imposed by the existence of only natural contrast between adjoining tissue types.

\section{Previous Work}

An algorithm, presented by Karssemeijer ${ }^{1}$ and referred to as "deterministic relaxation," has been developed for performing 3D segmentation of organs in abdominal CT scans.

$$
p\left(x_{i}=k \mid y, x_{S} \backslash i\right) \propto p\left(y_{i} \mid x_{i}\right) p\left(x_{i}=k\right)
$$

An application of Bayes' Theorem, this algorithm is based on the assumption that the probability that a certain voxel $i$ is labeled $k$ given the set of scene densities $y$ and the labeling of neighboring voxels $x_{S \backslash i}$ is proportional to the product of a) the probability that the voxel $i$ has a certain intensity $y_{i}$ given its labeling $x_{i}$ and $b$ ) the probability of that voxel having a certain labeling $k$. By iterating this relation over the set of voxels in the scene, the algorithm relaxes the scene to a steady state segmentation.

The algorithm makes the following calculation for the $p\left(x_{i}=K\right)$ term of (1):

$$
p\left(x_{i}=K\right) \leftarrow e^{\left[\mathcal{A}_{i}(\mathcal{K})-\sum_{n=1}^{K}\left(\mathcal{B}(K, n) g_{i}(n)\right)\right]}
$$

Here, $\mathscr{A}_{i}(K)$ is an external field and is approximated by $\ln \left(p\left(x_{i}=K\right)\right), \mathcal{B}(K, n)$ is a scalar representing the interaction between regions labeled $k$ and $n$, and $g_{i}(n)$ is a count of the neighboring voxels which are labeled $n$.

\section{Adaptation to Myocardial CT Scans}

A few changes have been made to the algorithm to make it more applicable to the task of segmenting myocardial CT scans. First, Karssemeijer's algorithm involves $3 \mathrm{D}$ segmentation. In our endeavor, we have focused on improving the segmentation technique in $2 \mathrm{D}$, believing that it can be easily extended to 3D.

Second, since the $A_{i}(K)$ is approximated by $\ln \left(p\left(x_{i}=K\right)\right.$, the term $p\left(x_{i}=\mathcal{K}\right)$ can be taken out and moved in front of the exponent. This is shown below where $z$ represents the summation term in (2).

$$
e^{\left[\ln \left(p\left(x_{i}=k\right)\right)-Z\right]}=p\left(x_{i}=k\right) e^{-Z}
$$

The interaction parameters used in Karssemeijer's implementation range from $+\infty$ to $-\infty$, are symmetric (i.e. $\mathcal{B}(i, j)=\mathcal{B}(j, i))$, and appear to be empirically determined. In an attempt to automate the generation of the interaction parameters, values are generated according to the percentage of the collective boundary of regions labeled $k$ which also lie on the boundaries of regions labeled $n$. Based on this approach, the interaction parameters range from zero to unity.

Another interesting result of this scheme for generating values for the interaction parameters is that the values are not symmetric. For example, consider 
the regions in Figure 1.

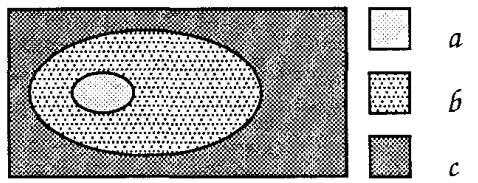

Figure 1. Sample Tissue Boundaries

$\mathcal{B}(a, b)$ is defined to be the percentage of the boundary of region $a$ which also bounds region 6 and is obviously $100 \%$ since region $a$ is enveloped by region 6 . On the other hand, $\mathcal{B}(b, a)$ - the percentage of the boundary of $b$ which also bounds $a-$ is only $20 \%$. Thus, $B(a, b) \neq$ $\mathcal{B}(b, a)$.

Third, the $g_{i}(n)$ term (the count of the number of neighbors of voxel $i$ which are labeled $n$ ) has been replaced by a scalar (lying between zero and unity) representing the percentage of the neighboring pixels which are labeled $n$. This way, the biasing effect of having fewer neighbors (such as along the edges and in the corners of the scene) are normalized.

Fourth, in order to approximate a probability for $p\left(x_{i=k}\right)$ between zero and unity, Karssemeijer's original equation is further changed so that the reciprocal of the summation (of the products of the interaction parameters and the neighborhood percentages) is used. The resulting modification for (2) is as follows:

$$
p\left(x_{i}=k\right) \leftarrow p\left(x_{i}=k\right) e^{\left[-\frac{1}{\sum_{n=1}^{k}\left(\mathcal{B}(\mathcal{K} n)_{g_{i}}(n)\right)}\right]}
$$

Hence, the entire segmentation equation is shown below:

$$
p\left(x_{i}=K\right) \leftarrow p\left(y_{i} \mid x_{i}=K\right) p\left(x_{i}=K\right)_{e}\left[-\frac{1}{\sum_{n=1}^{K}\left(\mathcal{B}(k, n) g_{i}(n)\right)}\right]_{(5)}
$$

\section{Implementation}

The implementation is comprised of four modules: manual segmentation, training set construction, calculation of a priori probabilities, and automated segmentation.

A set of mid-heart, end-diastole Cine CT scans are manually-segmented by painting into an overlay buffer. A set of manually-segmented images comprise a training set from which a set of initial a priori probabilities are calculated for position and density.
These probabilities are used to seed the iteration equation (5) in the automated segmentation module. The resulting segmentation can be folded back into the system by adding it to the training set. With such a mechanism, the system becomes dynamic and can learn from each segmentation.

Interaction parameters, in this implementation, are static. For each of six tissue classes, a row of scalar values are determined which represent the percentage of its boundary which also bounds regions of each of the other tissue classes. The interaction parameters are

\begin{tabular}{|c|c|c|c|c|c|c|}
\hline & $\mathrm{a}$ & $\mathrm{b}$ & c & $\mathrm{d}$ & $\mathrm{e}$ & $\bar{f}$ \\
\hline \multicolumn{7}{|l|}{$k$} \\
\hline a & 1.00 & 0.60 & 0.01 & 0.07 & 0.07 & 0.25 \\
\hline b & 0.85 & 1.00 & 0.02 & 0.10 & 0.01 & 0.02 \\
\hline c & 0.01 & 0.01 & 1.00 & 0.45 & 0.45 & 0.08 \\
\hline $\mathrm{d}$ & 0.30 & 0.10 & 0.50 & 1.00 & 0.01 & 0.09 \\
\hline $\mathrm{e}$ & 0.30 & 0.01 & 0.40 & 0.01 & 1.00 & 0.28 \\
\hline $\mathrm{f}$ & 0.25 & 0.05 & 0.20 & 0.20 & 0.30 & 1.00 \\
\hline \multicolumn{7}{|c|}{$\sim$ Key } \\
\hline Symbol & \multicolumn{2}{|c|}{ Tissue Class } & Symbol & \multicolumn{3}{|c|}{ Tissue Class } \\
\hline $\bar{a}$ & \multicolumn{2}{|c|}{ Myocardial Tissue } & $\mathrm{d}$ & \multicolumn{3}{|c|}{ Interstitial Tissue } \\
\hline$b$ & \multirow{2}{*}{\multicolumn{2}{|c|}{ Blood }} & e & \multicolumn{3}{|c|}{ Miscellaneous Tissue } \\
\hline c & & & $\mathrm{f}$ & & Lung $\mathrm{Ti}$ & \\
\hline
\end{tabular}
shown in Figure 2.

Figure 2. Interaction Parameter Table

The subprocess of using the system to automatically segment images is depicted in Figure 3.

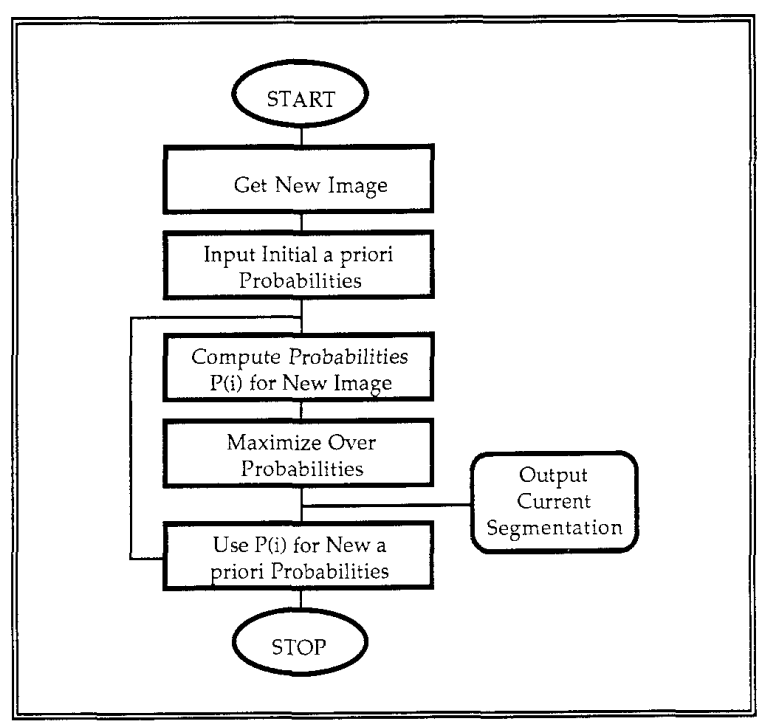

Figure 3. Automated Segmentation Process 
The process begins by obtaining a new image. A set of a priori probabilities, previously calculated from the current training set, are introduced to the system and used to seed the iteration equation (5). The iteration loop begins by computing a new set of probabilities $\mathrm{P}(\mathrm{i})$ as a function of the new image and the a priori probabilities. For each pixel, the set of probabilities for the contending tissue classes is maximized and a current segmentation is displayed. The previous $a$ priori probabilities, initially seeded with statistics gathered from the training set, are replaced by the new set of probabilities $\mathrm{P}(\mathrm{i})$ and the iteration loop is started anew. Upon conformance to convergence criteria, the iteration loop is terminated and a final segmentation is output.

\section{Special Techniques}

During the course of experimentation with this system, certain deficiencies became apparent. This section discusses some techniques employed to fortify the algorithm. First, an arbitrary set of CT scans will show a range of densities for the same regions of interest - particularly in regions enhanced with artificial contrast. Since density distributions are a critical factor in the segmentation algorithm, normalizing densities becomes desirable. Two regions which should have consistent densities in all pathologies are the lung (air) and bone. By establishing a norm $\mathcal{N}$ for these two tissue classes, any pathology $Q$ can be normalized according to a linear ramp based on these norms, clipping to minimum and maximum intensities where applicable, as shown below:

$$
Q_{i} \leftarrow\left(\frac{\mathcal{N}_{\text {Gone }}-\mathcal{N}_{\text {air }}}{Q_{\text {bone }}-Q_{\text {air }}}\right) \times\left(Q_{i}-Q_{\text {air }}\right)+\mathcal{N}_{\text {air }}
$$

Second, the algorithm is strongly dependant upon alignment of regions of interest within each scan. While isolating myocardial regions is the primary goal of this investigation, consistency in the relative position of these regions across various pathologies is low. However, some degree of consistency can be achieved by anchoring a square to fiduciary points of the pathology. The center of the spinal canal defines the lower-left corner of the square while the top of the heart wall specifies the top of the square.

Third, the scale of the image, in terms of pixels, must be the same in all images. While differing pathologies will naturally exhibit a range of sizes, some sizenormalization scheme must be applied. Bilinear interpolation seems a logical choice. However, such a technique can introduce density artifacts which have in practice resulted in misclassifications along tissue boundaries. An alternate approach is to subsample the original image. This technique is guaranteed to not introduce any new densities into the spatially normalized image.
Fourth, for a given pixel $i$, there exists a set of new $a$ priori position probabilities (one for each tissue class $k$ that describes the likelihood that $i$ should be labeled $k$ ). Early testing revealed that new a priori position probabilities generated during the first iteration of the automated segmentation process are highly polarized and do not allow for much change (relaxation) in subsequent iterations. If the polarity of these probabilities could be reduced, there would be greater opportunity for migration of tissue class boundaries. A technique has been employed which ranks the newlycalculated probabilities and maps new values to them. To the tissue class with the highest probability, the new value of 0.5 is assigned. To the tissue class with the second highest probability, the new value of 0.25 is assigned. This continues through all tissue classes, each successive position in rank receiving half the value of its predecessor. This new assignment scheme has three merits: First, it maintains the relative position of the probabilities (thus resulting in the same segmentation). Second, it is mathematically pure since, as the number of of tissue classes goes to infinity, the sum of all newly-assigned probabilities is unity. Third, it allows those tissue classes whose probabilities have been polarized to the low end of the scale an opportunity to participate in the subsequent iteration.

\section{$\underline{\text { Results }}$}

In order to validate the system, a number of tests were performed, varying the size of the training set and automatically segmenting both an image from the training set and one not from the training set. Each iteration of the segmentation was performed on a $64 \times 64$ image and required 3 minutes to process.

\section{Limited Training Set Applied to Training Set Image}

A limited training set, composed of four scans from each of two pathologies for a total of eight scans, was applied to one of the scans from the training set. Figure 4 shows the initial a priori position probabilities for the myocardial tissue and blood pool.

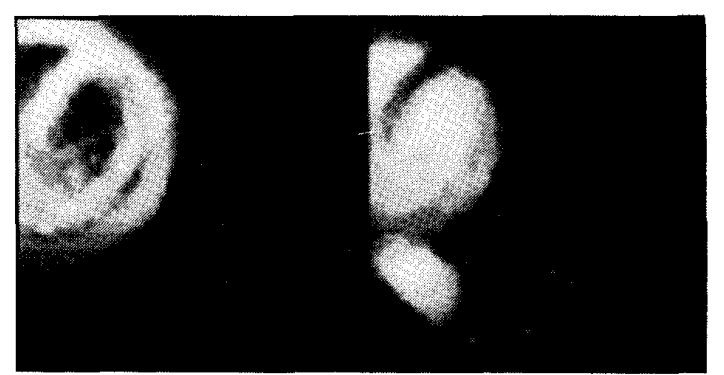

Fígure 4. Limited Training Set Probabilities 
The effect on the myocardial tissue probabilities of the first three iterations of the segmentation can be seen in Figure 5. Only modest change can be seen after the second iteration.

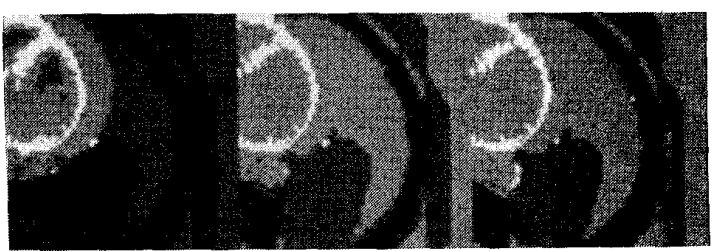

Figure 5. Position Probabilities from Three Iterations of Segmentation with Limited Training Set

\section{Extended Training Set Applied to Training Set Image}

By extended the training set to include a total of eighteen scans from five pathologies, the segmentation process was applied to the same image as before. Figure 6 depicts the pair of initial a priori position probabilities for the myocardial tissue and blood pool which are considerably more diffuse than those shown previously in Figure 4 for the limited training set.

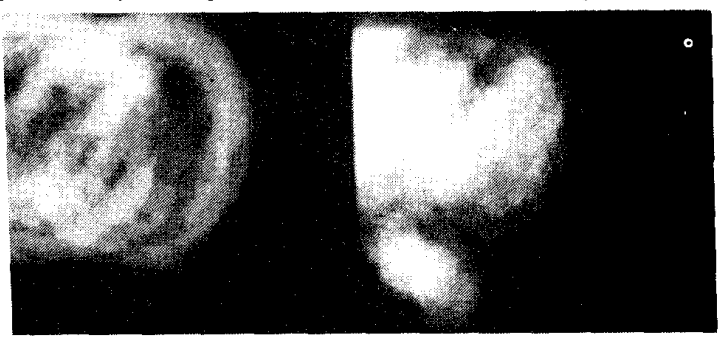

Figure 6. Extended Training Set Probabilities

The results of segmenting the image against the extended training set were slightly more accurate in fewer iterations than those produced by the limited training set.

\section{Extended Training Set Applied to Test Image}

Using the same extended training set as before, segmentation was applied to an image not found in the training set. The effect on the myocardial tissue probabilities of the first three iterations of the segmentation are shown in Figure 7 . Of particular interest is the improving definition of the myocardium, especially in the septal and posterior walls.

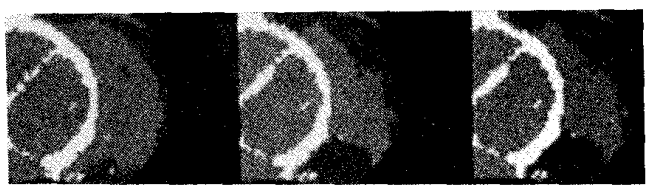

Figure 7. Position Probabilities from Three Iterations of Segmentation with Extended Training Set

\section{Conclusions}

Based on the results presented in the previous section as well as other tests applied to the system, the following conclusions are drawn. First, the probabilistic model works well for segmentation of myocardial tissue. Second, extending the training set (even to the inclusion of aneurysms and other abnormal pathologies) actually improves segmentation performance in terms of accuracy and the number of iterations required - contrary to initial expectations. Third, using an extensible training set provides the means for folding in new results so that the system can learn - from the addition of automated as well as manual segmentations.

\section{Future Work}

Experience with the current implementation has brought to light a few areas which could be enhanced. First, the spatial and density normalization functions could be automated. The current implementation requires user intervention with a mouse. Second, using image-to-image functions, supported in hardware by many image processing systems, could reduce the time required per iteration. Third, based on the encouraging results obtained when extending the training set from eight to eighteen, further extension of the training set to include a large number of images may afford more flexible relaxation between successive iterations. Fourth, a more heuristic approach to remapping probabilities during the iteration process may yield more consistent segmentation behavior.

\section{References}

1. Karssemeijer, N. Three-Dimensional Stochastic Organ-Models for Segmentation in CT-Scans. SPIE vol. 1030. Biostereometrics '88, Basel, Switzerland, 1988. 